\title{
Weight loss practices among newly enrolling clients in a commercial weight loss program in Ghana
}

\author{
Sandra Ayisi-Addo ${ }^{1}$, Stephen Ayisi-Addo ${ }^{1,2}$ and Agartha Ohemeng ${ }^{1}$ \\ ${ }^{1}$ Nutriline Research Centre, P.O Box KB 464, Korle-bu, Accra, ${ }^{2}$ Ghana National AIDS/STI Control Pro- \\ gramme, Accra, Ghana
}

DOI: $h t t p: / / d x$.doi.org/10.4314/gmj.v50i1.6

Corresponding author: Mrs Sandra Ayisi-Addo E-mail: nutriline20@yahoo.com

Conflict of interest: None declared

\section{SUMMARY}

Background: In Ghana, obesity is showing a rising trend and there are weight loss initiatives being practised by individuals. However, the levels of commitment to such programs and the reasons for discontinuing have not been assessed. The objectives of this study were to investigate the weight loss practices of participants and reasons for quitting chosen weight loss programs.

Methods: This was a cross-sectional study involving 50 subjects conveniently selected from people who were enrolling into a commercial weight loss program. A questionnaire was used to collect data on past weight loss practices and reasons for abandoning chosen programs. Data analysis was conducted using simple frequency and descriptive tests of the Excel software.

Results: More than half of the subjects (66\%) had undertaken a weight loss practice before enrolment in study. Of these, $88.5 \%$ abandoned the strategy before the achievement of their desired weight goal. The three common programs that were practised were internet based diets $(67.9 \%)$, commercial weight loss shakes $(42.9 \%)$ and exercises (28.6\%). Reasons given for abandoning programs included lack of sustainability $(50 \%)$, lack of determination on the part of the client (15\%), boredom with program (10\%), not achieving desired results $(10 \%)$, safety concerns $(10 \%)$, and getting pregnant $(5 \%)$.

Conclusions: The findings suggest that future weight loss programs can be effective and have reduced attrition rates if they are designed to achieve sustainable dietary and other lifestyle changes, as well as boost motivation for weight loss.

Keywords: weight loss, practices, diet, exercises, Ghana.

\section{INTRODUCTION}

Obesity is defined as a condition of abnormal or excessive accumulation of body fat usually exceeding $25 \%$ for men and $35 \%$ for women, to the extent that health is impaired. ${ }^{1,2}$ The World Health Organization has defined obesity as a disease because of the numerous health risks associated with it. ${ }^{2}$ These include type II diabetes, hypertension, high blood cholesterol, gout, heart attack, stroke, sleep apnoea, and premature death. World-wide, there is an increasing prevalence in obesity with about 2.8 million people dying yearly from obesity related conditions. ${ }^{1}$ The rising prevalence of obesity can be attributed largely to environmental factors such as poor dietary habits and sedentary lifestyle although other factors like genes also play a role. ${ }^{3}$ Once considered a problem only in high income countries, overweight and obesity are now dramatically on the rise in low- and middle-income countries, particularly in urban settings. Currently, these countries account for $80 \%$ of deaths from non communicable diseases, most of which are comorbidities associated with obesity. ${ }^{4}$ Among Ghanaian women of reproductive age, there has been a rise in the prevalence of overweight and obesity from $25 \%$ in 2003 to $30 \%$ in $2008 .{ }^{5,6}$. There are however regional differences in the prevalence, with the highest occurring in the Greater Accra region (42\%) among adults 18 years old and above. ${ }^{7}$. Obesity is also more prevalent among females and urban dwellers. ${ }^{5,7-9}$.

Weight loss aims to prevent weight gain, reduce weight, or maintain a healthy body weight and weight loss has been associated with improved health. Weight loss strategies include dietary therapy, physical activity, lifestyle modifications, pharmacotherapy, and occasionally surgery. ${ }^{10}$ General awareness on obesity and nutrition is increasing among Ghanaians and there is a public demand for lasting solutions to their weight loss needs. 
In response to this increased interest, there are public and private programs in the country designed to provide weight loss education or services. These include the national 'regenerative health' campaign spearheaded by the Ghana Health Service to promote healthy eating and physical activity. ${ }^{11}$. There are also increased training of dieticians to offer weight loss services in government and private health centers, meal replacement based commercial programs, internet- and TV-based weight loss programs, non-clinic type private weight loss centres, and gyms. There is however no documentation of the usage of these strategies. To strengthen existing programs and guide the development of new ones, there is the need for information on existing weight loss practices and reasons for attrition among Ghanaians.

The objective of this study was therefore to elucidate information on the weight loss practices and reasons why methods were abandoned before the achievement of desired weight goal from clients of a commercial weight loss program.

\section{METHODS}

Nutriline is a privately-owned company in Accra, Ghana with the mission of improving the health of the public through the provision of quality nutrition and weight loss services to both individuals and corporate bodies. This was a cross-sectional study of new clients enrolling in the Nutriline weight loss programme and the willingness to complete the study questionnaire was the criterion for inclusion into study. A total of 50 clients were consecutively selected between October and November 2012. A verbal consent was sought from all clients during enrollment and study participants could withdraw from the study at any stage of questionnaire administration.

A pre-tested questionnaire was used to collect data on demography, the weight loss methods that had previously been practiced before enrolling in Nutriline, whether they abandoned the program or not before achieving their desired weight goal, and reasons for abandoning the method if they did. Weight loss method was defined as any effort made in an attempt to lose weight and included commercial weight loss programs, exercises, meal replacements programs like Forever Living and Herbal Life, diet programs either learnt from dieting literature or the internet, drugs, any substance like teas, laxatives, used to try to lose weight or actually lose weight before enrolment into the study. These were not pre-listed for subjects to choose from, they had to list these methods by themselves.
Anthropometric (weight and height) measurements were also taken. Responses to questions were coded and the Body Mass Index (BMI) was calculated by dividing the weight by the square of the height measured in metres. All data were entered and analysed using simple frequency and descriptive tests in Microsoft Excel.

\section{RESULTS}

\section{Demography and anthropometry}

The mean age of the subjects was $37.6 \pm 10.4$ years and more than half of them $(56 \%)$ below the age of 40 years. Majority of them $(86 \%)$ were females (Table 1). The educational level of the study subjects was high, with $82 \%$ of them having more than secondary level education. The study sample was mainly obese (Table 1) with a mean BMI of $33.3 \pm 6.6 \mathrm{~kg} / \mathrm{m}^{2}$. More than half $(66 \%)$ were obese, and almost a third (28\%) were overweight. Only three subjects had a normal BMI at the time of enrolling in the Nutriline program with the aim of maintaining a healthy weight.

Table 1 Anthropometric parameters of study participants $^{1}$

\begin{tabular}{|l|l|l|}
\hline & $\begin{array}{l}\text { Males } \\
(\mathbf{n}=\mathbf{7})\end{array}$ & $\begin{array}{l}\text { Females } \\
(\mathbf{n}=\mathbf{4 3})\end{array}$ \\
\hline Age (y) & $44.1(12.2)$ & $36.6(9.8)$ \\
\hline Weight $(\mathrm{kg})$ & $103.7(26.1)$ & $88.9(18.4)$ \\
\hline Height $(\mathrm{m})$ & $1.75(0.06)$ & $1.65(0.07)$ \\
\hline $\mathrm{BMI}^{2}\left(\mathrm{~kg} / \mathrm{m}^{2}\right)$ & $33.6(7.5)$ & $33.2(6.6)$ \\
\hline Overweight (\%) & 42.9 & 25.6 \\
\hline Obese (\%) & 57.1 & 67.4 \\
\hline
\end{tabular}

\section{Weight loss practices of participants}

Majority of the subjects (66\%) had participated in a weight loss strategy in an attempt to lose prior to enrolment in the study. The three main weight loss methods followed in the past by participants were internet based programs $(67.9 \%)$, commercial shake-based programs such as Herbal Life and Forever Living (42.9\%), and exercises (28.6\%) (Figure 1) Internet-based strategies included low carbohydrate diet, Atkins diet, lemonade diet, blood group diet, food combination diet, apple cider diet, cabbage soup diet, and Cambridge diet. Of those who had used a weight loss method prior to enrolment in the study, $57.1 \%$ reported that their participation in those programs yielded some results during the period that they practised it. However, majority $(88.5 \%)$ of the subjects abandoned the program before the achievement of their desired weight goals and only $11.5 \%$ followed through with program. 


\section{Original Article}

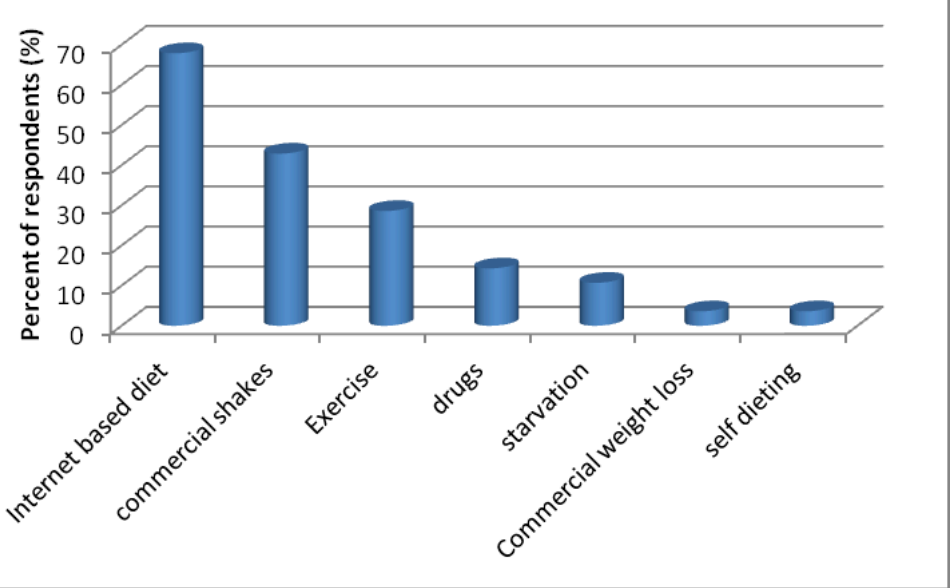

Figure 1:The weight loss strategies used by participants prior to enrollment into Nutriline program

Several reasons were given for abandoning weight loss programs prior to the achievement of goals (Figure 2). Half of the subjects reported abandoning program because it lacked sustainability. They could not for instance stay off carbohydrates for a long time. Lack of determination was another reason for drop-out (15\%) from weight loss programs and $10 \%$ reported quitting because they were rather gaining weight instead of losing.

Figure 2 Reasons for not completing previous weight loss strategies in Accra, Ghana

The other reasons participants gave for not completing programs included getting fed-up with the diet (10\%),

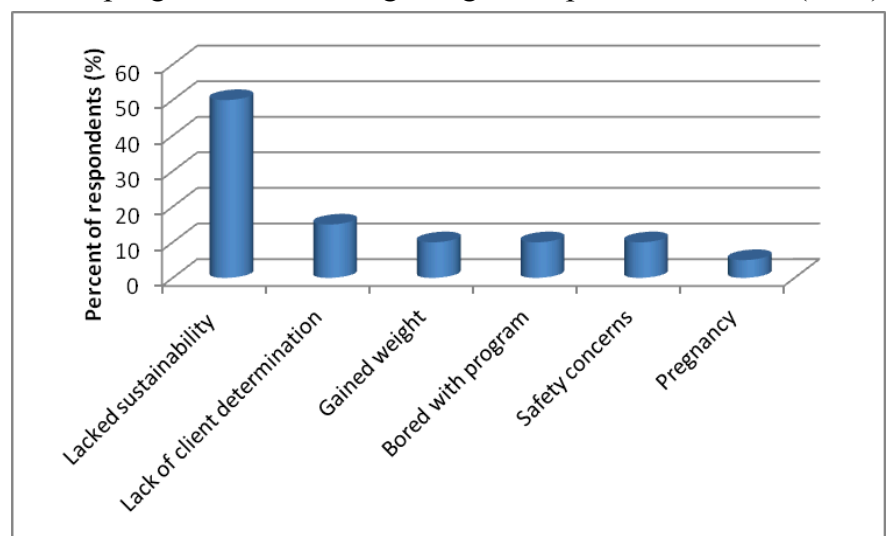

being afraid of side effects (10\%), delivering a baby and as such the timing not being right $(5 \%)$.

\section{DISCUSSION}

Majority of subjects in this study were females giving credence to the suggestion that females are more likely participate in weight loss programs more than men, similar to findings from earlier studies. In the United States, more females than males reported that they had tried to lose weight during the previous 12 months. ${ }^{12,13}$ Similarly, the assessment of a web-based weight loss program in Australia showed that $89 \%$ of prescribers were females. ${ }^{14}$ This high level of participation in weight loss programs by women may be a reflection of factors such as the high level of obesity among females, a greater concern for health, and increased societal pressure on women to be thin. ${ }^{15,16}$ Three studies that looked at obesity among adults in Ghana showed higher prevalence of both overweight and obesity among women when compared to men. ${ }^{7,8,17}$ Additionally, women may be more concerned with their health and well-being and want to take actions in this respect. Among obese patients seeking treatment in Italian medical centres, the two main motivations for weight loss were concern for health problems and women's desire to improve their appearance. ${ }^{18}$ Among Ghanaian women aged 18 years and above who resided in the Accra Metropolitan area, $72 \%$ were dissatisfied with their current body weight and $41.8 \%$ preferred a smaller body size. ${ }^{9}$ The study also reported that overweight and obese women were more likely to desire weight loss compared to normal weight women. Similarly, majority of women who were attending clinic at the Korle-Bu Teaching Hospital indicated that they would reduce their current body weight to achieve an overall healthier life. ${ }^{19}$ Interestingly, 63\% of the women stated that they would change their body weight if requested by the spouse. These studies show the high level of dissatisfaction among obese women with regards to body weight, which may then motivate them to attempt various weight loss strategies.

With the exception of three subjects who were normal weight, the rest of subjects were either overweight or obese despite the fact that majority of them had previously participated in a weight loss program before. This is not surprising, considering that our study observed a high attrition rate $(88.5 \%)$ among participants for previous weight loss programs. This raises questions about the effectiveness of the available weight loss programs and/or the ability of clients to follow their chosen weight loss methods correctly and through to the end. The observed attrition rate is higher than that reported by Dalle et al. , but it is comparable to rates observed in other studies in Italy and Australia. 14, 20, 21, 22 Majority of our study subjects were youthful, below the age of 40 years and may have been much younger when they engaged in the previous weight loss methods. 
Previous studies indicate that people of younger age are more likely to drop out of weight loss programs and this may partly explain the high attrition rate for previous weight loss programs reported by subjects in our study. ${ }^{14,20,23,24}$

Most of the weight loss programs observed in this study were diet or exercised based. This agrees with earlier studies in the United States of America and Brazil which reported diet and exercise as the weight loss practises most frequently used. ${ }^{12,13,25,26}$

Fundamentally, overweight and obesity reflects positive energy balance (WHO factsheet), and physical activity and dietary habits are two components of this balance that can be modified in a population. The use of drugs and starvation as methods used to lose weight need further investigation to assess how widespread these practices are. Although certain drugs are accepted for use as part of a holistic treatment for obesity in cases of morbid obesity, the safety of the drug is of concern and professional management is very important. Starvation or skipping meals was found to be one of the less common practices as found in a previous study. ${ }^{13}$ Starvation is not an approved method of weight loss as it is detrimental to the health of the individual. There are possibilities of nutrient deficiencies, low energy intakes with resultant hypoglycaemia, slowing down of the body's metabolism, overeating tendencies at the next meal and other negative side effects which come as a result of skipping meals or starving oneself.

No mention was made of using the conventional dietetic professionals available at private and public hospitals. Most of the commonly used strategies reported in this study were unlikely to be supervised by professionals with the exception of exercise programs. The lack of professional involvement in these programs may partly account for the high attrition and failure rates. The lack of professional guidance can result in overweight and obese subjects having unrealistic weight loss expectations at the beginning of a program, a factor that has been associated with high attrition rates. ${ }^{18,} 20$ This is because when these unrealistic goals are not met, subjects become dissatisfied and de-motivated to continue with a program. ${ }^{21}$ Overweight and obese individuals need education on setting realistic weight loss goals and the health benefits associated with just a $5-10 \%$ reduction in body weight, a goal that is achievable and sustainable. ${ }^{27,28}$ Programs will be well accepted and followed through until the achievement of desired weight goal if they provide realistic diet plans that are more practical to the client in terms of closeness to their original dietary habits, are interesting, results oriented within a reasonable time length, safe, and motivates the client to continue in the program. ${ }^{29}$ Subjects who get pregnant while on a weight loss program need professional guidance to enable them to switch from weight loss to weight management to ensure the attainment of the right pregnancy weight at term. This will help reduce risk of pregnancy complications that come as a result of excessive weight gain during pregnancy.

The limitations of this study are the use of a small sample size sampling only from enrolling clients of a commercial weight loss programme. Study results cannot be generalised to the general Ghanaian population due to the sampling style which made the results skewed to that of weight conscious individuals.

\section{Conclusion}

This study identified internet-based diet programs, commercial shake-based programs such as Herbal Life and Forever Living, and exercises as the common weight loss methods that were previously practised by subjects who enrolled in the Nutriline weight loss program. Although majority of weight conscious individuals pursued a weight loss method, they abandoned the method before the achievement of their desired weight goal. Future weight loss programs must focus on making weight loss program more practical to ensure sustainability and need to include the professional supervision provided by nutritionists and dieticians. A cross sectional population study with larger sample size that also assesses factors that are associated with the choice of weight loss methods and drop-out from weight loss programs will help give better insight into the weight loss practices of the general Ghanaian population.

\section{ACKNOWLEDGEMENT}

The authors wish to express their sincere thanks to Mr. Fui Acolatse for data entry and summaries of data inputs, as well as to all the clients who participated in the study.

\section{REFERENCES}

1. WHO.

http:www.who.int/mediacentre/factsheet/fs311/en/in dex.html Accessed on April 30, 2013.

2. WHO. Obesity: Preventing and managing the global epidemic. WHO Technical Report Series 894. Geneva 2000.

3. Kiranmala N, Das M, Arora N. Determinants of Childhood Obesity: Need for a Trans-Sectoral Convergent 2013;80(Suppl 1):S38-S47.

4. Shetty P. Nutrition Transition and Its Health Outcomes. Indian J Pediatr 2013;80(Supp11):S21-S7. 
5. Ghana Statistical Service (GSS), Ghana Health Service (GHS) and ICF Macro Ghana Demographic and Health Survey 2008. Accra, Ghana: GSS, GHS, and ICF Macro, 2009.

6. Ghana Statistical Service (GSS), Noguchi Memorial Institute for Medical Research (NMIMR), and ORC Macro Ghana Demographic and Health Survey 2003. Calverton, Maryland: GSS, NMIMR, and ORC Macro, 2004.

7. Biritwum R, Gyapong J, Mensah G. The Epidemiology of Obesity in Ghana. Ghana Med $J$ 2005;39(3):82-5.

8. Amoah A. Obesity in adult residents in Accra, Ghana. Ethn Dis 2003;13(Suppl 2):S97-S101.

9. Benkeser R, Biritwum R, Hill A. Prevalence of overweight and obesity and perception of healthy and desirable body size in urban, Ghanaian women. Ghana Med J 2012;46(2):66-75.

10. Ofei F. Obesity - A Preventive Disease. Ghana Med J 2005;39(3):98-101.

11. Ghana Health Service. Progress Report of the Regenerative Health and Nutrition Project. Accra, Ghana 2007.

12. Federation of American Societies for Experimental Biology. Third Report on Nutrition Monitoring in the United States: Executive Summary.Washington, DC 1995.

13. Weiss EC, Galuska DA, Khan LK, Serdula MK. Weight-Control Practices Among U.S. Adults, 2001-2002. Am J Prev Med 2006;31(1):18-24.

14. Neve M, Collins C, Morgan P. Nonusage Attrition, and Pre-treatment Predictors of Nonusage Attrition in a Commercial-Based Weight Loss Program. $J$ Med Internet Res 2010;12(4):e69.

15. Bayyari WD, Henry LJ, Jones C. Dieting behaviours, obesity and predictors of dieting among female college students at Palestinian universities. East Mediterr Health J 2013;19(1):30-6.

16. Fernandez S, Pritchard M. Relationships between self-esteem, media influence and drive for thinness. Eat Behav 2012;13:321-5.

17. Addo J, Smeeth L, Leon D. Obesity in urban civil servants in Ghana: Association with pre-adult wealth and adult socio-economic status. Public Health 2009; 123(5):365-70

18. Dalle Grave R, Calugi S, Magri F, Cuzzolaro M, Dall'Aglio E, Lucchin L, et al. Weight Loss Ex- pectations in Obese Patients Seeking Treatment at Medical Centers. Obes Res 2004;12(12):2005-12.

19. Duda R, Jumah N, Hill A, Seffah J, Biritwum R. Interest in healthy living outweighs presumed cultural norms for obesity for Ghanaian women. Health Qual Life Outcomes 2006;4:44-50.

20. Dalle Grave R, Calugi S, Molinari E, Petroni ML, Bondi M, Compare A, et al. Weight Loss Expectations in Obese Patients and Treatment Attrition: An Observational Multicenter Study. Obes Res 2005;13(11):1961-9.

21. Grossi E, Dalle Grave R, Mannucci E, Molinari E, Compare A, Cuzzolaro M, et al. Complexity of attrition in the treatment of obesity: clues from a structured telephone interview. Int $J$ Obes 2006;30:1132-7.

22. Inelmen E, Toffanello E, Enzi G, Gasparini G, Miotto F, Sergi G, et al. Predictors of drop-out in overweight and obese outpatients. Int $J$ Obes 2005;29:122-8.

23. Fabricatore AN, Wadden TA, Moore RH, Butryn ML, Heymsfield SB, Nguyen AM. Predictors of attrition and weight loss success: Results from a randomized controlled trial. Behav Res Ther 2009;47(8):685-91.

24. Gill R, Karmali S, Hadi G, Al-Adra D, Shi X, Birch D. Predictors of attrition in a multidisciplinary weight management clinic. Can J Surg 2012;55(4):239-43.

25. James DC. Weight loss strategies used by African American women: possible implications for tailored messages. J Hum Nutr Diet 2013;26(1):71-7.

26. Machado EC, Silveira MF, Silveira VM Prevalence of weight-loss strategies and use of substances for weight-loss among adults: a population study. Cad Saude Publica 2012;28(8):1439-49.

27. Blackburn G. Effect of degree of weight loss on health benefits. Obes Res 1995;3(Suppl 2):211S-6S.

28. Goldstein D. Beneficial health effects of modest weight loss. Int $J$ Obes Relat Metab Disord 1992;16:397-415.

29. Grafenauer S, Tapsell L, Beck E, Batterham M. Baseline dietary patterns are a significant consideration in correcting dietary exposure for weight loss. Eur J Clin Nutr 2013;67:330-6. 